\title{
SPECTRUM OF THE EXPONENTS OF BEST RATIONAL APPROXIMATION
}

\author{
DAMIEN ROY
}

\begin{abstract}
Using the new theory of W. M. Schmidt and L. Summerer called parametric geometry of numbers, we show that the going-up and going-down transference inequalities of W. M. Schmidt and M. Laurent describe the full spectrum of the $n$ exponents of best rational approximation to points in $\mathbb{R}^{n+1}$.
\end{abstract}

\section{INTRODUCTION}

The study of simultaneous rational approximation to points in $\mathbb{R}^{n}$ started with the basic estimates of Dirichlet in [2], and got full impetus in the years 1926-1938 through the transference theorems of Khintchine [5, 6] and Járnik [4]. Around the same period of time, Mahler introduced new tools in geometry of numbers and applied them to these questions [9]. In 1967, Schmidt [11] enlarged the scope of the problem by studying how a fixed vector subspace $A$ of $\mathbb{R}^{n}$ or $\mathbb{C}^{n}$ can be approximated, in the ambient space, by vector subspaces of a given dimension, defined over a fixed number field $K$. For this purpose, he introduced the notion of height of such a subspace and was lead to study several angles of approximation depending on the dimension of $A$. This important work was recently revisited by Laurent [8] in the case where $A$ is a one-dimensional subspace of $\mathbb{R}^{n}$ and where $K=\mathbb{Q}$ is the field of rational numbers. Then, $A$ is spanned by a single vector $\mathbf{u}$ and there is only one angle of approximation to consider. This lead Laurent to introduce a family of $n-1$ (ordinary) exponents of approximation to points in $\mathbb{R}^{n}$ (interpolating between the two classical ones) and to recast the results of Schmidt in that setting through a series of inequalities relating these exponents. The purpose of this paper is to show that these inequalities describe the full spectrum of these exponents, thus answering a question of Laurent in [8]. The proof uses the parametric geometry of numbers introduced by Schmidt and Summerer in a series of recent papers [12, 13], together with the complements from [10].

In the next section, we recall the definition of these exponents as well as the inequalities of Schmidt and Laurent which link them, and we state our main result. In Section 3 , we express these exponents in the context of parametric geometry of numbers. In Section 4 , we introduce the notion of generalized $n$-system and, using [10], we reduce the proof of our main result to a combinatorial problem involving such systems. We study a particular family of generalized $n$-systems in Section 5 and use them in Section 6 to complete the proof.

2010 Mathematics Subject Classification. 11J13.

Work partially supported by NSERC. 


\section{Notation AND MAin RESUlt}

For each integer $n \geq 1$, we view $\mathbb{R}^{n}$ as an Euclidean space for the standard inner product of two vectors $\mathbf{x}$ and $\mathbf{y}$ denoted $\mathbf{x} \cdot \mathbf{y}$. We also view its exterior algebra $\bigwedge \mathbb{R}^{n}=\oplus_{k=0}^{n} \bigwedge^{k} \mathbb{R}^{n}$ as an Euclidean space characterized by the property that, for each orthonormal basis $\left\{\mathbf{e}_{1}, \ldots, \mathbf{e}_{n}\right\}$ of $\mathbb{R}^{n}$, the products $\mathbf{e}_{i_{1}} \wedge \cdots \wedge \mathbf{e}_{i_{k}}$ with $0 \leq k \leq n$ and $1 \leq i_{1}<\cdots<i_{k} \leq n$ form an orthonormal basis of $\bigwedge \mathbb{R}^{n}$. For each $k=1, \ldots, n$, we also denote by $\bigwedge^{k} \mathbb{Z}^{n}$ the lattice of $\bigwedge^{k} \mathbb{R}^{n}$ spanned by the products $\mathbf{x}_{1} \wedge \cdots \wedge \mathbf{x}_{k}$ with $\mathbf{x}_{1}, \ldots, \mathbf{x}_{k} \in \mathbb{Z}^{n}$. For $k=0$, we have $\bigwedge^{k} \mathbb{R}^{n}=\mathbb{R}$ and we set $\bigwedge^{0} \mathbb{Z}^{n}=\mathbb{Z}$.

We say that a vector subspace $S$ of $\mathbb{R}^{n}$ is defined over $\mathbb{Q}$ if it is generated over $\mathbb{R}$ by elements of $\mathbb{Q}^{n}$. For such a subspace $S$, a basis $\left\{\mathbf{x}_{1}, \ldots, \mathbf{x}_{k}\right\}$ of $S \cap \mathbb{Z}^{n}$ as a $\mathbb{Z}$-module is also a basis of $S$ as a vector space over $\mathbb{R}$ and, following Schmidt [11, we define the height of $S$ by

$$
H(S)=\left\|\mathbf{x}_{1} \wedge \cdots \wedge \mathbf{x}_{k}\right\|
$$

this being independent of the choice of $\left\{\mathbf{x}_{1}, \ldots, \mathbf{x}_{k}\right\}$.

Given a non-zero point $\mathbf{u}$ in $\mathbb{R}^{n}$ and a non-zero subspace $S$ of $\mathbb{R}^{n}$, we define the projective distance between $\mathbf{u}$ and $S$ by

$$
\operatorname{dist}(\mathbf{u}, S)=\frac{\left\|\mathbf{u} \wedge \mathbf{x}_{1} \wedge \cdots \wedge \mathbf{x}_{k}\right\|}{\|\mathbf{u}\|\left\|\mathbf{x}_{1} \wedge \cdots \wedge \mathbf{x}_{k}\right\|}
$$

where $\left\{\mathbf{x}_{1}, \ldots, \mathbf{x}_{k}\right\}$ is any basis of $S$ over $\mathbb{R}$. Again this is independent of the choice of the basis. It is also given by

$$
\operatorname{dist}(\mathbf{u}, S)=\frac{\left\|\operatorname{proj}_{S^{\perp}}(\mathbf{u})\right\|}{\|\mathbf{u}\|}
$$

where $\operatorname{proj}_{S^{\perp}}(\mathbf{u})$ denotes the orthogonal projection of $\mathbf{u}$ on the orthogonal complement $S^{\perp}$ of $S$. Geometrically, it represents the sine of the smallest angle between $\mathbf{u}$ and a non-zero vector of $S$. In the work of Schmidt [11, this quantity is denoted $\psi_{1}(A, S)$ or $\omega_{1}(A, S)$ where $A=\mathbb{R} \mathbf{u}$ is the line spanned by $\mathbf{u}$.

Definition 2.1. Let $n \geq 1$ and let $\mathbf{u} \in \mathbb{R}^{n+1} \backslash\{0\}$. For each $j=0, \ldots, n-1$, we denote by $\omega_{j}(\mathbf{u})$ (resp. $\left.\hat{\omega}_{j}(\mathbf{u})\right)$ the supremum of all real numbers $\omega$ such that, for arbitrarily large values of $Q$ (resp. for all sufficiently large values of $Q$ ), there exists a vector subspace $S$ of $\mathbb{R}^{n+1}$, defined over $\mathbb{Q}$, of dimension $j+1$, with

$$
H(S) \leq Q \quad \text { and } \quad H(S) \operatorname{dist}(\mathbf{u}, S) \leq Q^{-\omega} .
$$

In particular, we have $\omega_{j}(\mathbf{u})=\infty$ if $\mathbf{u}$ belongs to a subspace $S$ of $\mathbb{R}^{n+1}$ defined over $\mathbb{Q}$ of dimension $j+1$. Otherwise, $\omega_{j}(\mathbf{u})$ is the supremum of all real numbers $\omega$ for which there exist infinitely many subspaces $S$ of $\mathbb{R}^{n+1}$, defined over $\mathbb{Q}$, of dimension $j+1$ with

$$
\operatorname{dist}(\mathbf{u}, S) \leq H(S)^{-\omega-1} \text {. }
$$

Theorem 13 of [11] shows that

$$
\omega_{j}(\mathbf{u}) \geq \hat{\omega}_{j}(\mathbf{u}) \geq \frac{j+1}{n-j} \quad(0 \leq j \leq n-1) .
$$

Thus $\omega_{0}(\mathbf{u}), \ldots, \omega_{n-1}(\mathbf{u})$ are non-negative although possibly infinite. The following result provides further inequalities relating these exponents of approximation. 
Theorem 2.2 (Schmidt, Laurent). Let $n \in \mathbb{N}^{*}$. For any non-zero $\mathbf{u} \in \mathbb{R}^{n+1}$, we have $\omega_{0}(\mathbf{u}) \geq 1 / n$ and

$$
\frac{j \omega_{j}(\mathbf{u})}{\omega_{j}(\mathbf{u})+j+1} \leq \omega_{j-1}(\mathbf{u}) \leq \frac{(n-j) \omega_{j}(\mathbf{u})-1}{n-j+1} \quad(1 \leq j \leq n-1),
$$

with the convention that the left-most ratio is equal to $j$ if $\omega_{j}(\mathbf{u})=\infty$.

The left inequality in (2.2) follows from the Going-up-theorem of Schmidt [11, Theorem 9] while the right inequality follows from his Going-down-theorem [11, Theorem 10]. This was observed by Laurent in [8] who also introduced the exponents $\omega_{j}(\mathbf{u})$ for that purpose. In the same paper, Laurent also notes that each individual inequality in (2.2) is best possible because their combination yields Khinchine's transference inequalities which are known to be best possible. He also deduces that, for each fixed $j \in\{0, \ldots, n-1\}$, the spectrum of $\omega_{j}$, namely the set of all possible values $\omega_{j}(\mathbf{u})$, is the full interval $[(j+1) /(n-j), \infty]$. Although he restricts to points $\mathbf{u}$ with $\mathbb{Q}$-linearly independent coordinates, this is also true over the the set of all $\mathbf{u} \neq 0$ in view of (2.1).

An independent proof for the right inequality in (2.2) is given by Laurent in [8] and, for both inequalities, by Bugeaud and Laurent in [1], again for points $\mathbf{u}$ with $\mathbb{Q}$-linearly independent coordinates.

Our main result below answers a question of Laurent in [8] by showing that the inequalities of Theorem 2.2 describe the set of all possible values for the $n$-tuples $\left(\omega_{0}(\mathbf{u}), \ldots, \omega_{n-1}(\mathbf{u})\right)$.

Theorem 2.3. Let $n \in \mathbb{N}^{*}$. For any $\omega_{0}, \ldots, \omega_{n-1} \in[0, \infty]$ satisfying $\omega_{0} \geq 1 / n$ and

$$
\frac{j \omega_{j}}{\omega_{j}+j+1} \leq \omega_{j-1} \leq \frac{(n-j) \omega_{j}-1}{n-j+1} \quad(1 \leq j \leq n-1),
$$

there exists a point $\mathbf{u} \in \mathbb{R}^{n+1}$ with $\mathbb{Q}$-linearly independent coordinates such that

$$
\omega_{j}(\mathbf{u})=\omega_{j} \quad \text { and } \quad \hat{\omega}_{j}(\mathbf{u})=\frac{j+1}{n-j} \quad(0 \leq j \leq n-1) .
$$

A description of the spectrum of the $2 n$ exponents $\left(\omega_{0}, \ldots, \omega_{n-1}, \hat{\omega}_{0}, \ldots, \hat{\omega}_{n-1}\right)$ was achieved by Laurent in [7] for $n=2$ but, for larger values of $n$, the question is open. In [13, §1], Schmidt and Summerer propose a different larger set of $2 n+2$ exponents of approximation to non-zero points in $\mathbb{R}^{n}$. Their spectrum also is unknown, even for $n=2$.

\section{LiNK WITH PARAMETRIC GEOMETRY OF NUMBERS}

Fix $n \in \mathbb{N}^{*}$ and $\mathbf{u} \in \mathbb{R}^{n+1} \backslash\{0\}$. For each real number $Q \geq 1$, we form the convex body

$$
\mathcal{C}_{\mathbf{u}}(Q)=\left\{\mathbf{x} \in \mathbb{R}^{n+1} ;\|\mathbf{x}\| \leq 1,|\mathbf{x} \cdot \mathbf{u}| \leq Q^{-1}\right\}
$$

and, for $j=1, \ldots, n+1$, we denote by $\lambda_{j}\left(\mathcal{C}_{\mathbf{u}}(Q)\right)$ its $j$-th minimum, namely the smallest real number $\lambda>0$ such that $\lambda \mathcal{C}_{\mathbf{u}}(Q)$ contains at least $j$ linearly independent points of $\mathbb{Z}^{n+1}$. On the model of Schmidt and Summerer in [13], we define

$$
L_{\mathbf{u}, j}(q)=\log \lambda_{j}\left(\mathcal{C}_{\mathbf{u}}\left(e^{q}\right)\right) \quad(q \geq 0,1 \leq j \leq n+1),
$$

and we form the map $\mathbf{L}_{\mathbf{u}}:[0, \infty) \rightarrow \mathbb{R}^{n+1}$ given by

$$
\mathbf{L}_{\mathbf{u}}(q)=\left(L_{\mathbf{u}, 1}(q), \ldots, L_{\mathbf{u}, n+1}(q)\right) \quad(q \geq 0) .
$$


For each $j=1, \ldots, n+1$, we also define

$$
\underline{\psi}_{j}(\mathbf{u})=\liminf _{q \rightarrow \infty} \frac{L_{\mathbf{u}, 1}(q)+\cdots+L_{\mathbf{u}, j}(q)}{q} \text { and } \quad \bar{\psi}_{j}(\mathbf{u})=\limsup _{q \rightarrow \infty} \frac{L_{\mathbf{u}, 1}(q)+\cdots+L_{\mathbf{u}, j}(q)}{q} .
$$

The following result connects these quantities to those from the previous section.

Proposition 3.1. Let $n$ and $\mathbf{u}$ be as above. For each $j \in\{0, \ldots, n-1\}$, we have

$$
\omega_{j}(\mathbf{u})=\frac{1}{\underline{\psi}_{n-j}(\mathbf{u})}-1 \quad \text { and } \quad \hat{\omega}_{j}(\mathbf{u})=\frac{1}{\bar{\psi}_{n-j}(\mathbf{u})}-1 .
$$

The proof relies on the following alternative definition for the exponents $\omega_{j}(\mathbf{u})$ and $\hat{\omega}_{j}(\mathbf{u})$.

Lemma 3.2 (Bugeaud-Laurent). Let $j \in\{0, \ldots, n-1\}$. Then $\omega_{j}(\mathbf{u})\left(\operatorname{resp} . \hat{\omega}_{j}(\mathbf{u})\right)$ is the supremum of all real numbers $\omega$ such that the inequalities

$$
\|\mathbf{z}\| \leq Q \quad \text { and } \quad\|\mathbf{z} \wedge \mathbf{u}\| \leq Q^{-\omega}
$$

have a non-zero solution $\mathbf{z} \in \bigwedge^{j+1} \mathbb{Z}^{n+1}$ for arbitrarily large values of $Q$ (resp. for all sufficiently large values of $Q$ ).

This is proved in [1, §4] for $\omega_{j}(\mathbf{u})$ in the case where $\mathbf{u}$ has $\mathbb{Q}$-linearly independent coordinates but the same argument extends with little change to any non-zero vector $\mathbf{u}$ and applies also to $\hat{\omega}_{j}(\mathbf{u})$.

Proof of Proposition 3.1. For each $j=0, \ldots, n-1$ and each $Q \geq 1$, define

$$
\mathcal{K}_{\mathbf{u}}^{(j+1)}(Q)=\left\{\mathbf{z} \in \bigwedge^{j+1} \mathbb{R}^{n+1} ;\|\mathbf{z}\| \leq Q,\|\mathbf{z} \wedge \mathbf{u}\| \leq 1\right\} .
$$

According to [1, $\S 4$, Lemma 3], $\mathcal{K}_{\mathbf{u}}^{(j+1)}(Q)$ is comparable to the $(j+1)$-th compound body of $\mathcal{K}_{\mathbf{u}}^{(1)}(Q)$. As the latter is in turn comparable to the dual of $\mathcal{C}_{\mathbf{u}}(Q)$ and as $\operatorname{vol}\left(\mathcal{C}_{\mathbf{u}}(Q)\right) \asymp Q^{-1}$, it follows that the first minimum of $\mathcal{K}_{\mathbf{u}}^{(j+1)}(Q)$ with respect to $\bigwedge^{j+1} \mathbb{Z}^{n+1}$ satisfies

$$
\begin{aligned}
\lambda_{1}\left(\mathcal{K}_{\mathbf{u}}^{(j+1)}(Q)\right) & \asymp \lambda_{1}\left(\mathcal{K}_{\mathbf{u}}^{(1)}(Q)\right) \cdots \lambda_{j+1}\left(\mathcal{K}_{\mathbf{u}}^{(1)}(Q)\right) \\
& \asymp \lambda_{n+1}\left(\mathcal{C}_{\mathbf{u}}(Q)\right)^{-1} \cdots \lambda_{n-j+1}\left(\mathcal{C}_{\mathbf{u}}(Q)\right)^{-1} \\
& \asymp Q^{-1} \lambda_{1}\left(\mathcal{C}_{\mathbf{u}}(Q)\right) \cdots \lambda_{n-j}\left(\mathcal{C}_{\mathbf{u}}(Q)\right) \quad(0 \leq j \leq n-1, Q \geq 1),
\end{aligned}
$$

with implied constants depending only on $n$ and $\mathbf{u}$ (see [3, $\S \S 14-15]$ ). Now the convex body of $\bigwedge^{j+1} \mathbb{R}^{n+1}$ defined by the conditions (3.1) is $Q^{-\omega} \mathcal{K}_{\mathbf{u}}^{(j+1)}\left(Q^{\omega+1}\right)$, so its first minimum with respect to $\bigwedge^{j+1} \mathbb{Z}^{n+1}$ is bounded below and above by products of

$$
Q^{-1} \lambda_{1}\left(\mathcal{C}_{\mathbf{u}}\left(Q^{\omega+1}\right)\right) \cdots \lambda_{n-j}\left(\mathcal{C}_{\mathbf{u}}\left(Q^{\omega+1}\right)\right)
$$

with positive constants that are independent of $Q$ and of $\omega$, when $Q \geq 1$ and $\omega+1 \geq 0$. By Lemma 3.2, $\omega_{j}(\mathbf{u})$ (resp. $\hat{\omega}_{j}(\mathbf{u})$ ) is the supremum of all real numbers $\omega$ for which this first minimum is $\leq 1$ for arbitrarily large values of $Q$ (resp. for all sufficiently large values of $Q$ ). Since $\omega_{j}(\mathbf{u}) \geq \hat{\omega}_{j}(\mathbf{u}) \geq 0$ by (2.1), we may restrict to values of $\omega$ with $\omega>-1$ and thus $\omega_{j}(\mathbf{u})$ (resp. $\hat{\omega}_{j}(\mathbf{u})$ ) is also the supremum of all real numbers $\omega>-1$ for which the product in (3.2) is $\leq 1$ for arbitrarily large values of $Q$ (resp. for all sufficiently large values 
of $Q)$. However, upon writing $Q=e^{q /(\omega+1)}$ with $q>0$, the condition that this product is $\leq 1$ amounts to

$$
\frac{L_{\mathbf{u}, 1}(q)+\cdots+L_{\mathbf{u}, n-j}(q)}{q} \leq \frac{1}{\omega+1},
$$

and the conclusion follows.

\section{Generalized $n$-Systems}

Fix an integer $n \geq 2$. In [13, §3], Schmidt and Summerer propose the notion of $(n, 0)$ system as a model for the general behavior of the maps $\mathbf{L}_{\mathbf{u}}:[0, \infty) \rightarrow \mathbb{R}^{n}$ attached to non-zero points $\mathbf{u} \in \mathbb{R}^{n}$. The following is a version of this adapted to the present context.

Definition 4.1. Let $I$ be a subinterval of $[0, \infty)$ with non-empty interior. An $n$-system on $I$ is a continuous piecewise linear map $\mathbf{P}=\left(P_{1}, \ldots, P_{n}\right): I \rightarrow \mathbb{R}^{n}$ with the following properties.

(S1) For each $q \in I$, we have $0 \leq P_{1}(q) \leq \cdots \leq P_{n}(q)$ and $P_{1}(q)+\cdots+P_{n}(q)=q$.

(S2) If $H$ is a non-empty open subinterval of $I$ on which $\mathbf{P}$ is differentiable, then there is an integer $r$ with $1 \leq r \leq n$ such that $P_{r}$ has slope 1 on $H$ while the other components $P_{j}$ of $\mathbf{P}$ with $j \neq r$ are constant on $H$.

(S3) If $q$ is an interior point of $I$ at which $\mathbf{P}$ is not differentiable and if the integers $r$ and $s$ for which $P_{r}^{\prime}\left(q^{-}\right)=P_{s}^{\prime}\left(q^{+}\right)=1$ satisfy $r<s$, then we have $P_{r}(q)=P_{r+1}(q)=\cdots=$ $P_{s}(q)$.

Here, the condition that $\mathbf{P}: I \rightarrow \mathbb{R}^{n}$ is piecewise linear means that the set $D$ of points of $I$ at which $\mathbf{P}$ is not differentiable (including the boundary points of $I$ that lie in $I$ ) is a discrete subset of $I$, and that the derivative of $\mathbf{P}$ is locally constant on $I \backslash D$. Such a map admits a left derivative $\mathbf{P}^{\prime}\left(q^{-}\right)$at each $q \in I$ with $q \neq \inf I$, and a right derivative $\mathbf{P}^{\prime}\left(q^{+}\right)$at each $q \in I$ with $q \neq \sup I$. The slope of a component $P_{r}$ of $\mathbf{P}$ on an open subinterval $H$ of $I \backslash D$ means the constant value of its derivative on $H$ or equivalently the slope of its graph over $H$.

Figure 1 shows the combined graph of the functions $P_{r}, \ldots, P_{s}$ over a neighborhood of $q$ under the hypotheses of Condition (S3). In that case, the functions $P_{r+1}, \ldots, P_{s}$ coincide to the left of $q$, while $P_{r}, \ldots, P_{s-1}$ coincide to its right.

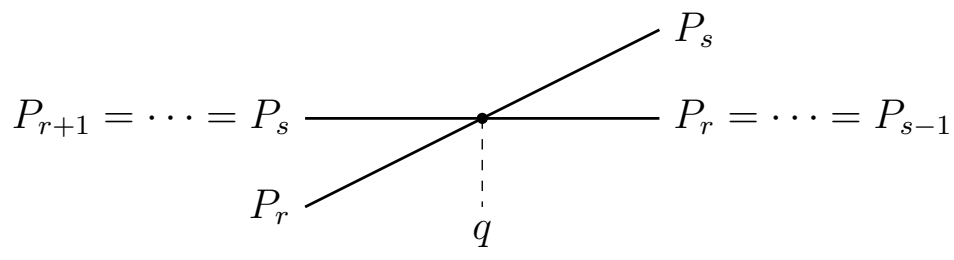

Figure 1. Illustration for Condition (S3).

For an interval $I$ of the form $\left[q_{0}, \infty\right)$ with $q_{0} \geq 0$, the above notion of an $n$-system on $I$ is the same as that of an $(n, 0)$-system on $I$ according to Definition 2.8 of [10]. These $n$-systems have the following approximation property. 
Theorem 4.2. For each non-zero point $\mathbf{u} \in \mathbb{R}^{n}$, there exists $q_{0} \geq 0$ and an n-system $\mathbf{P}$ on $\left[q_{0}, \infty\right)$ such that $\mathbf{L}_{\mathbf{u}}-\mathbf{P}$ is bounded on $\left[q_{0}, \infty\right)$. Conversely, for each n-system $\mathbf{P}$ on an interval $\left[q_{0}, \infty\right)$ with $q_{0} \geq 0$, there exists a non-zero point $\mathbf{u} \in \mathbb{R}^{n}$ such that $\mathbf{L}_{\mathbf{u}}-\mathbf{P}$ is bounded on $\left[q_{0}, \infty\right)$.

The first assertion follows from [10, Theorem 1.3] which states the same approximation property for a more restricted class of maps called rigid n-systems, while its converse follows from [10, Theorem 8.1]. The fact that we deal with non-zero points $\mathbf{u}$ of $\mathbb{R}^{n}$ instead of unit vectors like in [10] does not matter for such a qualitative statement.

The goal of this section is to show that this approximation property extends to the larger class of generalized $n$-systems which we will define below. In view of Proposition 3.1, this reduces the determination of the spectrum of any subsequence of $\left(\omega_{0}, \ldots, \omega_{n-1}, \hat{\omega}_{0}, \ldots, \hat{\omega}_{n-1}\right)$ over $\mathbb{R}^{n+1} \backslash\{0\}$ to a problem about generalized $(n+1)$-systems.

The next two lemmas provide examples of $n$-systems.

Lemma 4.3. Let $a, b \in \mathbb{R}$ with $0 \leq a<b$. There exists an n-system $\mathbf{P}:[a, b] \rightarrow \mathbb{R}^{n}$ such that $\mathbf{P}(a)=(a / n, \ldots, a / n)$ and $\mathbf{P}(b)=(b / n, \ldots, b / n)$.

Proof. Set $q_{i}=(n-i) a / n+i b / n$ for $i=0, \ldots, n$ and, for each $j=1, \ldots, n$, define $P_{j}$ to be the unique continuous piecewise linear function on $\left[q_{0}, q_{n}\right]=[a, b]$ which is constant equal to $a / n$ on $\left[q_{0}, q_{n-j}\right]$, has slope 1 on $\left[q_{n-j}, q_{n-j+1}\right]$, and is constant equal to $b / n$ on $\left[q_{n-j+1}, q_{n}\right]$. Then the map $\mathbf{P}=\left(P_{1}, \ldots, P_{n}\right):[a, b] \rightarrow \mathbb{R}^{n}$ is an $n$-system with the required properties. Its combined graph is illustrated on Figure 2 below.
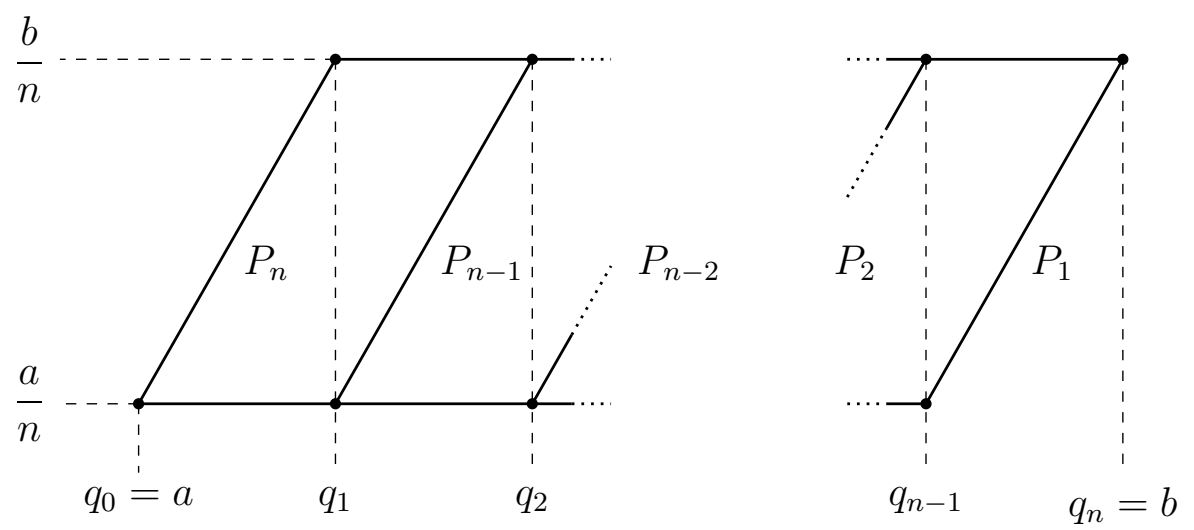

Figure 2. Combined graph of an $n$-system for Lemma 4.3 ,

Lemma 4.4. Let $a, b, c \in \mathbb{R}$ with $0 \leq a<b<c$. Suppose that $\mathbf{P}^{(1)}:[a, b] \rightarrow \mathbb{R}^{n}$ and $\mathbf{P}^{(2)}:[b, c] \rightarrow \mathbb{R}^{n}$ are $n$-systems with $\mathbf{P}^{(1)}(b)=\mathbf{P}^{(2)}(b)=(b / n, \ldots, b / n)$. Then there is an $n$-system $\mathbf{P}$ on $[a, c]$ which restricts to $\mathbf{P}^{(1)}$ on $[a, b]$ and to $\mathbf{P}^{(2)}$ on $[b, c]$.

Proof. There is a unique map $\mathbf{P}:[a, c] \rightarrow \mathbb{R}^{n}$ which restricts to $\mathbf{P}^{(1)}$ on $[a, b]$ and to $\mathbf{P}^{(2)}$ on $[b, c]$. It is continuous but not differentiable at $b$. However, it satisfies the condition (S3) at $q=b$ since all its coordinates are equal at that point. Thus, it is an $n$-system. 
Let $I$ be any subinterval of $[0, \infty)$ and let $D$ be an arbitrary discrete subset of $I$. By combining Lemmas 4.3 and 4.4, we can construct an $n$-system $\mathbf{P}$ on $I$ which takes the value $\mathbf{P}(q)=(q / n, \ldots, q / n)$ at each point $q$ of $D$. Then, by choosing $D$ appropriately, we may ensure that $\sup _{q \in I}\|\mathbf{P}(q)-(q / n, \ldots, q / n)\|_{\infty}$ is finite and arbitrarily small. As we will see, a slightly more general argument shows that these properties of approximation by $n$ systems extend to the following maps, besides the map $\tilde{P}: I \rightarrow \mathbb{R}^{n}$ sending each $q \in I$ to $(q / n, \ldots, q / n)$.

Definition 4.5. Let $I$ be a subinterval of $[0, \infty)$ with non-empty interior. A generalized $n$-system on $I$ is a continuous piecewise linear map $\mathbf{P}=\left(P_{1}, \ldots, P_{n}\right): I \rightarrow \mathbb{R}^{n}$ with the following properties.

(G1) For each $q \in I$, we have $0 \leq P_{1}(q) \leq \cdots \leq P_{n}(q)$ and $P_{1}(q)+\cdots+P_{n}(q)=q$.

(G2) If $H$ is a non-empty open subinterval of $I$ on which $\mathbf{P}$ is differentiable, then there are integers $\underline{r}, \bar{r}$ with $1 \leq \underline{r} \leq \bar{r} \leq n$ such that $P_{\underline{r}}, P_{\underline{r}+1}, \ldots, P_{\bar{r}}$ coincide on the whole interval $H$ and have slope $1 /(\bar{r}-\underline{r}+1)$, while any other component $P_{j}$ of $\mathbf{P}$ is constant on $H$.

(G3) If $q$ is an interior point of $I$ at which $\mathbf{P}$ is not differentiable, if $\underline{r}, \bar{r}, \underline{s}, \bar{s}$ are the integers for which

$$
P_{j}^{\prime}\left(q^{-}\right)=\frac{1}{\bar{r}-\underline{r}+1} \quad(\underline{r} \leq j \leq \bar{r}) \quad \text { and } \quad P_{j}^{\prime}\left(q^{+}\right)=\frac{1}{\bar{s}-\underline{s}+1} \quad(\underline{s} \leq j \leq \bar{s}),
$$

and if $\underline{r}<\bar{s}$, then we have $P_{\underline{r}}(q)=P_{\underline{r}+1}(q)=\cdots=P_{\bar{s}}(q)$.

Figure 3 shows the combined graph of the functions $P_{\underline{r}}, \ldots, P_{\bar{s}}$ over a neighborhood of $q$ under the hypotheses of (G3) when $\bar{r}<\underline{s}$ and $\bar{r}<\bar{s}$. The pattern is slightly different when $\underline{r} \geq \underline{s}$ (resp. when $\bar{r} \geq \bar{s}$ ): in that case, there is no horizontal line segment to the right of $q$ (resp. to the left of $q$ ). However, one cannot have $\underline{s} \leq \underline{r}<\bar{s} \leq \bar{r}$ and so at least one of the inequalities $\underline{r}<\underline{s}$ or $\bar{r}<\bar{s}$ must hold.

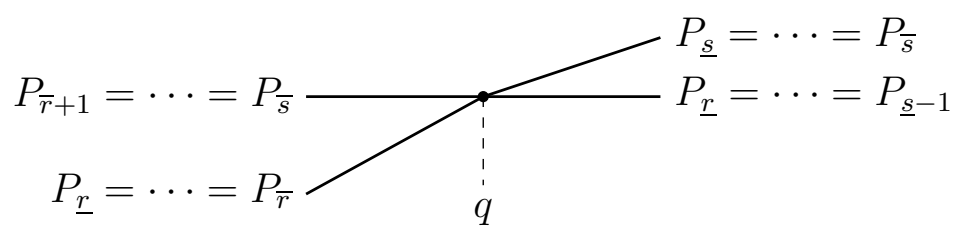

Figure 3. Illustration for Condition (G3) when $\bar{r}<\underline{s}$ and $\bar{r}<\bar{s}$.

Note also that if (4.1) holds at some point $q$ where $\mathbf{P}$ is not differentiable, and if $\underline{r} \geq \bar{s}$, then we must have $\underline{r}>\bar{s}$ and $P_{\underline{r}}(q)=\cdots=P_{\bar{r}}(q)>P_{\underline{s}}(q)=\cdots=P_{\bar{s}}(q)$. We leave the verification to the reader.

Clearly $n$-systems are also generalized $n$-systems. The next result and its corollary formalize the claims made just above Definition 4.5.

Proposition 4.6. Let $\tilde{\mathbf{P}}$ be a generalized $n$-system on a subinterval $I$ of $[0, \infty)$ with nonempty interior, and let $D$ be a discrete subset of $I$. Then there is an $n$-system $\mathbf{P}$ on $I$ such that $\mathbf{P}(t)=\tilde{\mathbf{P}}(t)$ for each $t \in D$. 
Proof. Let $D_{0}$ be the set of points of $I$ where $\tilde{\mathbf{P}}$ is not differentiable, including the boundary points of $I$ that lie in $I$ (if any). Since $D_{0}$ is a discrete subset of $I$, we may assume without loss of generality that $D$ contains $D_{0}$. We may also assume that $I$ and $D$ have the same infimum and the same supremum.

Let $\left(t_{1}, t_{2}\right)$ be a maximal subinterval of $I \backslash D$. Then, $t_{1}$ and $t_{2}$ belong to $D$ and, as $D \subseteq I$, it follows that $\left[t_{1}, t_{2}\right]$ is a subinterval of $I$. As $\tilde{\mathbf{P}}$ is differentiable on $\left(t_{1}, t_{2}\right)$, there are integers $\underline{r}$ and $\bar{r}$ with $1 \leq \underline{r} \leq \bar{r} \leq n$ such that $\tilde{P}_{\underline{r}}, \ldots, \tilde{P}_{\bar{r}}$ coincide and have slope $1 /(\bar{r}-\underline{r}+1)$ on $\left(t_{1}, t_{2}\right)$ while all other components $\tilde{P}_{j}$ are equal to a constant $c_{j}$ on that interval. Put

$$
m=\bar{r}-\underline{r}+1 \quad \text { and } \quad c=\left(c_{1}+\cdots+c_{\underline{r}-1}\right)+\left(c_{\bar{r}+1}+\cdots+c_{n}\right) .
$$

By Lemma 4.3, there exists an $m$-system $\left(A_{1}, \ldots, A_{m}\right)$ on $\left[t_{1}, t_{2}\right]$ with

$$
\left(A_{1}\left(t_{i}\right), \ldots, A_{m}\left(t_{i}\right)\right)=\left(\frac{t_{i}}{m}, \ldots, \frac{t_{i}}{m}\right) \quad \text { for } i=1,2 .
$$

Since $\tilde{P}_{j}(q)=(q-c) / m$ for each $j=\underline{r}, \ldots, \bar{r}$ and each $q \in\left[t_{1}, t_{2}\right]$, the map $\mathbf{P}:\left[t_{1}, t_{2}\right] \rightarrow \mathbb{R}^{n}$ given by

$$
\mathbf{P}(q)=\left(c_{1}, \ldots, c_{\underline{r}-1}, A_{1}(q)-\frac{c}{m}, \ldots, A_{m}(q)-\frac{c}{m}, c_{\bar{r}+1}, \ldots, c_{n}\right) \quad\left(t_{1} \leq q \leq t_{2}\right),
$$

coincides with $\tilde{\mathbf{P}}$ at the points $q=t_{1}, t_{2}$. As the intervals $\left[t_{1}, t_{2}\right]$ cover $I$ and have no interior point in common, this in fact defines a continuous piecewise linear map $\mathbf{P}: I \rightarrow \mathbb{R}^{n}$ which coincides with $\tilde{\mathbf{P}}$ on the set $D$.

To conclude, it remains simply to show that $\mathbf{P}$ satisfies the conditions (S1-S3) of Definition 4.1. For each $q$ in a fixed interval $\left[t_{1}, t_{2}\right]$, the formula (4.2) shows that the coordinates of $\mathbf{P}(q)$ sum up to $q$. They also form a monotone increasing sequence because $A_{1}(q) \leq \cdots \leq A_{m}(q)$ are separately monotone increasing functions of $q$ in $\left[t_{1}, t_{2}\right]$, and because the coordinates of $\mathbf{P}\left(t_{i}\right)=\tilde{\mathbf{P}}\left(t_{i}\right)$ form monotone increasing sequences for $i=1,2$. Thus the condition (S1) is fulfilled. The condition (S2) also holds by construction. The same is true for (S3) except possibly at the points $q$ of $D$ which lie in the interior of $I$. The latter points are the common boundary points $q=t_{2}$ of two maximal subintervals $\left(t_{1}, t_{2}\right)$ and $\left(t_{2}, t_{3}\right)$ of $I \backslash D$. Define $\underline{r}, \bar{r}$ as above and denote by $\underline{s}, \bar{s}$ the corresponding integers for the interval $\left[t_{2}, t_{3}\right]$. By construction, the function $\mathbf{P}$ satisfies $P_{\underline{r}}^{\prime}\left(t_{2}^{-}\right)=P_{\bar{s}}^{\prime}\left(t_{2}^{+}\right)=1$. Suppose that $\underline{r}<\bar{s}$. If $t_{2} \in D_{0}$, we have $\tilde{P}_{\underline{r}}\left(t_{2}\right)=\cdots=\tilde{P}_{\bar{s}}\left(t_{2}\right)$ because $\tilde{\mathbf{P}}$ satisfies the condition (G3) of Definition 4.5. If $t_{2} \notin D_{0}$, these equalities still hold because then $\underline{r}=\underline{s}$ and $\bar{r}=\bar{s}$. As $\mathbf{P}\left(t_{2}\right)=\tilde{\mathbf{P}}\left(t_{2}\right)$, we conclude that $P_{\underline{r}}\left(t_{2}\right)=\cdots=P_{\bar{s}}\left(t_{2}\right)$ and thus (S3) is satisfied.

Corollary 4.7. Let $\tilde{\mathbf{P}}: I \rightarrow \mathbb{R}^{n}$ be a generalized $n$-system and let $\epsilon>0$. Then there is an $n$-system $\mathbf{P}$ on $I$ such that $\|\tilde{\mathbf{P}}(q)-\mathbf{P}(q)\|_{\infty} \leq \epsilon$ for each $q \in I$.

Proof. Let $D$ be the set of all integer multiples of $\epsilon / 2$ in $I$. By Proposition 4.6, there is an $n$-system $\mathbf{P}$ on $I$ such that $\mathbf{P}(t)=\tilde{\mathbf{P}}(t)$ for each $t \in D$. Since the components of $\mathbf{P}$ and of $\tilde{\mathbf{P}}$ have slopes between 0 and 1 , we find that

$$
\|\tilde{\mathbf{P}}(q)-\mathbf{P}(q)\|_{\infty} \leq\|\tilde{\mathbf{P}}(q)-\tilde{\mathbf{P}}(t)\|_{\infty}+\|\mathbf{P}(q)-\mathbf{P}(t)\|_{\infty} \leq 2|q-t|
$$

for each $q \in I$ and each $t \in D$. Upon choosing $t$ so that $|q-t| \leq \epsilon / 2$, we conclude that $\mathbf{P}$ has the required property. 
Corollary 4.7 shows that the set $\mathcal{S}_{I}$ of $n$-systems on a subinterval $I$ of $[0, \infty)$ is dense in the set $\mathcal{G}_{I}$ of generalized $n$-systems on $I$, for the topology of uniform convergence. More precisely, it can be shown that $\mathcal{G}_{I}$ is the completion of $\mathcal{S}_{I}$ for that topology. This justifies working with functions from this set. In particular, the validity of Theorem 4.2 extends to generalized $n$-systems.

\section{A FAMILY OF GENERALIZED $n$-SYSTEMS}

Again, we fix an integer $n \geq 2$. Consider the set

$$
\Delta^{(n)}=\left\{\left(a_{1}, \ldots, a_{n}\right) \in \mathbb{R}^{n} ; 0<a_{1}<\cdots<a_{n} \text { and } a_{1}+\cdots+a_{n}=1\right\}
$$

and its topological closure

$$
\bar{\Delta}^{(n)}=\left\{\left(a_{1}, \ldots, a_{n}\right) \in \mathbb{R}^{n} ; 0 \leq a_{1} \leq \cdots \leq a_{n} \text { and } a_{1}+\cdots+a_{n}=1\right\} .
$$

By condition (G1) from Definition 4.5, we have $q^{-1} \mathbf{P}(q) \in \bar{\Delta}^{(n)}$ for any generalized $n$-system $\mathbf{P}$ and any $q>0$ in its interval of definition. For each $j=1, \ldots, n$, we define a map $\psi_{j}: \bar{\Delta}^{(n)} \rightarrow \mathbb{R}$ by

$$
\psi_{j}\left(a_{1}, \ldots, a_{n}\right)=a_{1}+\cdots+a_{j} .
$$

The proof of our main result relies on the following basic construction.

Proposition 5.1. Let $\mathbf{a}=\left(a_{1}, \ldots, a_{n}\right) \in \Delta^{(n)}$. Define

$$
\begin{aligned}
& q_{i}=a_{1}+\cdots+a_{i}+(n-i) a_{i} \quad(1 \leq i \leq n), \\
& q_{n-1+i}=(i-1) a_{i}+a_{i}+\cdots+a_{n} \quad(1 \leq i \leq n) .
\end{aligned}
$$

Then there exists a unique generalized n-system $\mathbf{P}=\left(P_{1}, \ldots, P_{n}\right)$ on $\left[q_{1}, q_{2 n-1}\right]=\left[n a_{1}, n a_{n}\right]$ whose combined graph is as shown on Figure 4 below. For each $j=1, \ldots, n-1$, it has

$$
\inf \psi_{j}\left(q^{-1} \mathbf{P}(q)\right)=\psi_{j}(\mathbf{a}) \quad \text { and } \quad \sup \psi_{j}\left(q^{-1} \mathbf{P}(q)\right)=\frac{j}{n}
$$

where both infimum and supremum are taken over all $q \in\left[n a_{1}, n a_{n}\right]$.

In Figure 4, the number $1 / m$ next to each slanted line segment indicates its slope and thus the number $m$ of components of $\mathbf{P}$ whose graph coincide with this line segment over the corresponding interval $\left[q_{i}, q_{i+1}\right]$.

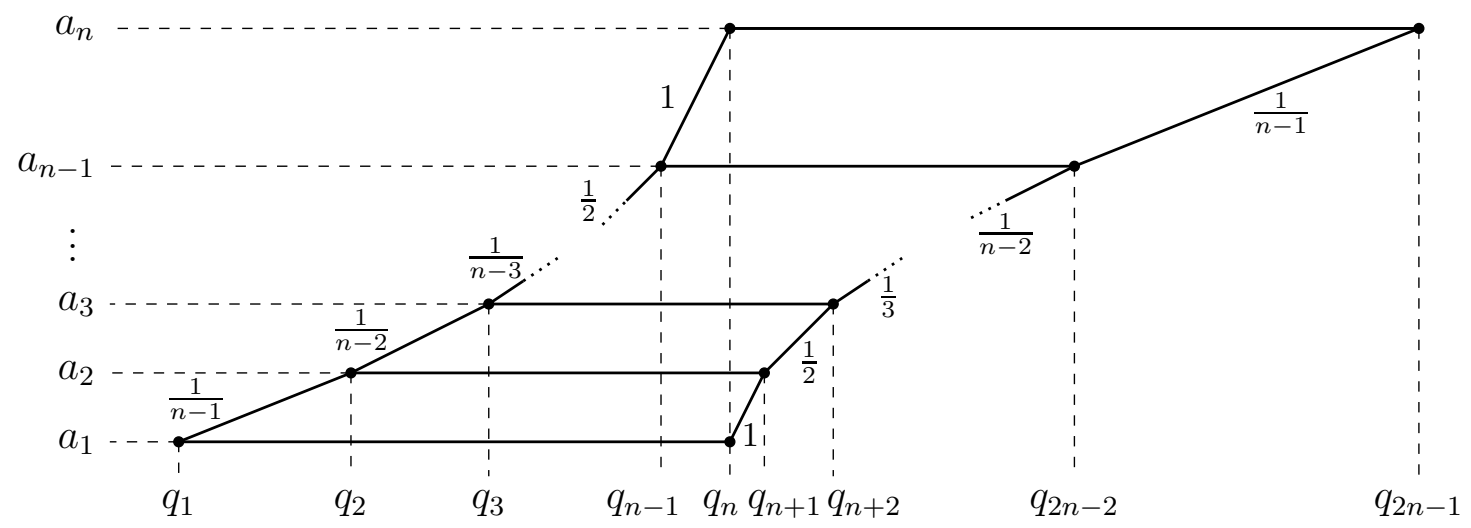

FigurE 4. The combined graph of a generalized $n$-system. 
The existence and uniqueness of $\mathbf{P}$ is easily seen. In order to compute the required infimum and supremum, we use the following result.

Lemma 5.2. Let $0<a<b$, let $M:[a, b] \rightarrow \mathbb{R}$ be a continuous piecewise linear function on $[a, b]$, let $q_{1}=a<q_{2}<\cdots<q_{t}=b$ be the points where $M$ is not differentiable (including $a$ and $b$ ), and let $\rho_{i}$ denote the constant value of the derivative of $M$ on $\left(q_{i}, q_{i+1}\right)$ for $i=1, \ldots, t-1$.

1) If $M(a) / a>\rho_{1}>\cdots>\rho_{t-1}$, then $M(q) / q$ is strictly decreasing on $[a, b]$, and bounded below by $\rho_{t-1}$.

2) If $\rho_{1}>\cdots>\rho_{t-1}>M(b) / b$, then $M(q) / q$ is strictly increasing on $[a, b]$, and bounded above by $\rho_{1}$.

Proof. It suffices to prove this for $t=2$ because the general case then follows by induction on $t$. Assuming that $t=2$, we can write

$$
M(q)=M(a)+\rho_{1}(q-a)=M(b)+\rho_{1}(q-b) \quad(a \leq q \leq b) .
$$

Under the hypothesis 1), we also have $M(a)>\rho_{1} a$ and so $M(q) / q=\rho_{1}+\left(M(a)-\rho_{1} a\right) / q$ is a strictly decreasing function of $q$ on $[a, b]$, bounded below by $\rho_{1}$. Similarly, under the hypothesis 2), we have $M(b)<\rho_{1} b$, then $M(q) / q=\rho_{1}+\left(M(b)-\rho_{1} b\right) / q$ is strictly increasing on $[a, b]$ and bounded above by $\rho_{1}$.

Proof of Proposition 5.1. As noted above, it suffices to prove the second assertion. To this end, fix an index $j$ with $1 \leq j \leq n-1$ and define $M_{j}=P_{1}+\cdots+P_{j}$. Then $M_{j}$ is a continuous piecewise linear map on $\left[q_{1}, q_{2 n-1}\right]$ with slope $(j-i) /(n-i)$ on $\left[q_{i}, q_{i+1}\right]$ for $i=1, \ldots, j-1$, slope 0 on $\left[q_{j}, q_{n}\right]$, slope 1 on $\left[q_{n}, q_{n+j}\right]$ and slope $j / i$ on $\left[q_{n+i-1}, q_{n+i}\right]$ for $i=j+1, \ldots, n-1$. Since

and

$$
\frac{M_{j}\left(q_{1}\right)}{q_{1}}=\frac{j}{n}>\frac{j-1}{n-1}>\cdots>\frac{1}{n-j+1}>0
$$

$$
1>\frac{j}{j+1}>\cdots>\frac{j}{n-1}>\frac{j}{n}=\frac{M_{j}\left(q_{2 n-1}\right)}{q_{2 n-1}},
$$

we conclude from Lemma 5.2 that the ratio $M_{j}(q) / q$ is strictly decreasing on $\left[q_{1}, q_{n}\right]$ and strictly increasing on $\left[q_{n}, q_{2 n-1}\right]$. This means that

$$
\inf \frac{M_{j}(q)}{q}=\frac{M_{j}\left(q_{n}\right)}{q_{n}}=\frac{a_{1}+\cdots+a_{j}}{a_{1}+\cdots+a_{n}}=\psi_{j}(\mathbf{a}) \quad \text { and } \quad \sup \frac{M_{j}(q)}{q}=\frac{j}{n} .
$$

The last result of this section combines the above proposition with the following observation.

Lemma 5.3. If $\mathbf{P}:[a, b] \rightarrow \mathbb{R}^{n}$ is a generalized $n$-system with $b>a>0$, then, for any $d>c>0$ with $d / c=b / a$, the map $\tilde{\mathbf{P}}:[c, d] \rightarrow \mathbb{R}^{n}$ given by

$$
\tilde{\mathbf{P}}(q)=\frac{c}{a} \mathbf{P}\left(\frac{a}{c} q\right) \quad \text { for each } q \in[c, d]
$$

is also a generalized $n$-system and we have both

$$
\inf _{q \in[a, b]} \psi_{j}\left(q^{-1} \mathbf{P}(q)\right)=\inf _{q \in[c, d]} \psi_{j}\left(q^{-1} \tilde{\mathbf{P}}(q)\right) \quad \text { and } \sup _{q \in[a, b]} \psi_{j}\left(q^{-1} \mathbf{P}(q)\right)=\sup _{q \in[c, d]} \psi_{j}\left(q^{-1} \tilde{\mathbf{P}}(q)\right) \text {. }
$$


The combined graph of this new map $\tilde{\mathbf{P}}$ is the image of the combined graph of $\mathbf{P}$ by uniform scaling of ratio $c / a$. Upon noting furthermore that Lemma 4.4 extends to generalized $n$-systems, we conclude with the following result.

Proposition 5.4. Let $E$ be an arbitrary non-empty subset of $\bar{\Delta}^{(n)}$. There exists a generalized $n$-system $\mathbf{P}$ on $[1, \infty)$ which, for each $j=1, \ldots, n$, satisfies

$$
\liminf _{q \rightarrow \infty} \psi_{j}\left(q^{-1} \mathbf{P}(q)\right)=\inf \psi_{j}(E) \quad \text { and } \quad \limsup _{q \rightarrow \infty} \psi_{j}\left(q^{-1} \mathbf{P}(q)\right)=\frac{j}{n} .
$$

Proof. If $E$ consists of the single point $(1 / n, \ldots, 1 / n)$, we simply take $\mathbf{P}(q)=(q / n, \ldots, q / n)$ for each $q \geq 1$. Otherwise, we choose a sequence $\left(\mathbf{a}^{(i)}\right)_{i \geq 1}$ of points of $\Delta^{(n)}$ whose set of accumulation points is the topological closure $\bar{E}$ of $E$. For each $i \geq 1$, we consider the generalized $n$-system attached to $\mathbf{a}^{(i)}$ by Proposition 5.1, and, starting with $q_{1}=1$, we use Lemma 5.3 recursively to transform it into a generalized $n$-system $\mathbf{P}^{(i)}$ on an interval of the form $\left[q_{i}, q_{i+1}\right]$. By construction, we have

$$
\inf _{q \in\left[q_{i}, q_{i+1}\right]} \psi_{j}\left(q^{-1} \mathbf{P}^{(i)}(q)\right)=\psi_{j}\left(\mathbf{a}^{(i)}\right) \quad \text { and } \quad \sup _{q \in\left[q_{i}, q_{i+1}\right]} \psi_{j}\left(q^{-1} \mathbf{P}^{(i)}(q)\right)=\frac{j}{n}
$$

for $j=1, \ldots, n$. We also note that

$$
\mathbf{P}^{(i-1)}\left(q_{i}\right)=\mathbf{P}^{(i)}\left(q_{i}\right)=\left(q_{i} / n, \ldots, q_{i} / n\right) \quad(i \geq 2)
$$

and that $\lim \sup q_{i+1} / q_{i}>1$ since $E$ contains at least one point $\left(a_{1}, \ldots, a_{n}\right)$ with $a_{n}>a_{1}$. Thus $\lim q_{i}=\infty$ and so there exists a unique generalized $n$-system $\mathbf{P}$ on $[1, \infty)$ which restricts to $\mathbf{P}^{(i)}$ on $\left[q_{i}, q_{i+1}\right]$ for each $i \geq 1$. In view of (5.1), we obtain

$$
\liminf _{q \rightarrow \infty} \psi_{j}\left(q^{-1} \mathbf{P}(q)\right)=\liminf _{q \rightarrow \infty} \psi_{j}\left(\mathbf{a}^{(i)}\right)=\inf \psi_{j}(E) \quad \text { and } \quad \limsup _{q \rightarrow \infty} \psi_{j}\left(q^{-1} \mathbf{P}(q)\right)=\frac{j}{n}
$$

for each $j=1, \ldots, n$, as requested.

\section{Proof of the main Result}

The next result is the last piece that we need in order to prove Theorem 2.3 .

Proposition 6.1. Let $n \in \mathbb{N}^{*}$. Suppose that $\underline{\psi}_{1}, \ldots, \underline{\psi}_{n} \in[0,1]$ satisfy $\underline{\psi}_{n} \leq n /(n+1)$,

$$
\frac{\underline{\psi}_{j}}{j} \leq \frac{\underline{\psi}_{j+1}}{j+1} \quad \text { and } \quad \frac{1-\underline{\psi}_{j}}{n+1-j} \leq \frac{1-\underline{\psi}_{j+1}}{n-j} \quad(1 \leq j \leq n-1) .
$$

Then, there exists a finite non-empty subset $E$ of $\bar{\Delta}^{(n+1)}$ such that

$$
\underline{\psi}_{j}=\min \psi_{j}(E) \quad(1 \leq j \leq n) .
$$

Proof. If $n=1$, we have $\underline{\psi}_{1} \leq 1 / 2$ and we simply take $E=\left\{\left(\underline{\psi}_{1}, 1-\underline{\psi}_{1}\right)\right\}$. Suppose from now on that $n \geq 2$ and let $k \in\{1, \ldots, n-1\}$. We claim that there exists $\mathbf{a} \in \bar{\Delta}^{(n+1)}$ such that $\psi_{j}(\mathbf{a}) \geq \underline{\psi}_{j}$ for $j=1, \ldots, n$, with equality for $j=k$ and $j=k+1$. To show this, set $c=\underline{\psi}_{k} / k$ and $d=\left(1-\underline{\psi}_{k+1}\right) /(n-k)$, and consider the point

$$
\mathbf{a}=(\overbrace{c, \ldots, c}^{k \text { times }}, \underline{\psi}_{k+1}-\underline{\psi}_{k}, \overbrace{d, \ldots, d}^{n-k \text { times }}) \in \mathbb{R}^{n+1} .
$$


By hypothesis, we have $c \geq 0$ and the inequalities (6.1) for $j=k$ yield $c \leq \underline{\psi}_{k+1}-\underline{\psi}_{k} \leq d$. As the sum of the coordinates of $\mathbf{a}$ is 1 , this means that $\mathbf{a} \in \bar{\Delta}^{(n+1)}$. We also note from (6.1) that the ratios $\underline{\psi}_{j} / j$ and $\left(1-\underline{\psi}_{j}\right) /(n+1-j)$ are both monotone increasing functions of $j$ for $j \in\{1, \ldots, n\}$. Thus, for $j=1, \ldots, k$, we have $\underline{\psi}_{j} / j \leq c$ and so we find $\psi_{j}(\mathbf{a})=j c \geq \underline{\psi}_{j}$, with equality if $j=k$. Similarly, for $j=k+1, \ldots, n$, we have $d \leq\left(1-\underline{\psi}_{j}\right) /(n+1-j)$ and so we obtain $\psi_{j}(\mathbf{a})=1-(n+1-j) d \geq \underline{\psi}_{j}$, with equality if $j=k+1$. This proves our claim. By varying $k$, this produces a finite set of points with the required property.

In general, we cannot expect that the set $E$ consists of a single element because each $\mathbf{a}=\left(a_{1}, \ldots, a_{n+1}\right)$ in $\bar{\Delta}^{(n+1)}$ satisfies

$$
\psi_{j-1}(\mathbf{a})+\psi_{j+1}(\mathbf{a})-2 \psi_{j}(\mathbf{a})=a_{j+1}-a_{j} \geq 0 \quad(2 \leq j \leq n),
$$

and so, for example, there is no point $\mathbf{a}$ in $\bar{\Delta}^{(4)}$ with $\psi_{1}(\mathbf{a})=0, \psi_{2}(\mathbf{a})=1 / 3$ and $\psi_{3}(\mathbf{a})=1 / 2$, although the numbers $\underline{\psi}_{1}=0, \underline{\psi}_{2}=1 / 3$ and $\underline{\psi}_{3}=1 / 2$ satisfy the conditions (6.1) for $n=3$.

Proof of Theorem [2.3. Suppose that $\omega_{0}, \ldots, \omega_{n-1} \in[0, \infty]$ satisfy the conditions of the theorem. Then the numbers

$$
\underline{\psi}_{j}=\frac{1}{\omega_{n-j}+1} \in[0,1] \quad(1 \leq j \leq n),
$$

fulfill the hypotheses of the above proposition. Let $E$ be a corresponding subset of $\bar{\Delta}^{(n+1)}$, as in that proposition, and let $\mathbf{P}$ be a generalized $(n+1)$-system on $[1, \infty)$ as constructed by Proposition 5.4 for this choice of $E$. For $j=1, \ldots, n$, we have

$$
\liminf _{q \rightarrow \infty} \psi_{j}\left(q^{-1} \mathbf{P}(q)\right)=\inf \psi_{j}(E)=\underline{\psi}_{j} \quad \text { and } \quad \limsup _{q \rightarrow \infty} \psi_{j}\left(q^{-1} \mathbf{P}(q)\right)=\frac{j}{n+1} .
$$

Corollary 4.7 and Theorem 4.2 provide a point $\mathbf{u} \in \mathbb{R}^{n+1} \backslash\{0\}$ for which the difference $\mathbf{P}-\mathbf{L}_{\mathbf{u}}$ is bounded on $[1, \infty)$. By the above, and by definition of the quantities $\underline{\psi}_{j}(\mathbf{u})$ and $\bar{\psi}_{j}(\mathbf{u})$ in Section 3, this point satisfies

$$
\underline{\psi}_{j}(\mathbf{u})=\underline{\psi}_{j} \quad \text { and } \quad \bar{\psi}_{j}(\mathbf{u})=\frac{j}{n+1} \quad(1 \leq j \leq n) .
$$

By Proposition 3.1, this means that, as requested, we have, for $j=0, \ldots, n-1$,

$$
\omega_{j}(\mathbf{u})=\frac{1}{\underline{\psi}_{n-j}(\mathbf{u})}-1=\omega_{j} \quad \text { and } \quad \hat{\omega}_{j}(\mathbf{u})=\frac{1}{\bar{\psi}_{n-j}(\mathbf{u})}-1=\frac{j+1}{n-j} .
$$

Since $\hat{\omega}_{n-1}(\mathbf{u})=n<\infty$, the coordinates of $\mathbf{u}$ must be linearly independent over $\mathbb{Q}$.

As a complement, note that each point $\mathbf{a}=\left(a_{1}, \ldots, a_{n+1}\right)$ in $\bar{\Delta}^{(n+1)}$ satisfies

$$
0 \leq \frac{\psi_{j}(\mathbf{a})}{j} \leq \frac{\psi_{j+1}(\mathbf{a})}{j+1} \quad \text { and } \quad 0 \leq \frac{1-\psi_{j}(\mathbf{a})}{n+1-j} \leq \frac{1-\psi_{j+1}(\mathbf{a})}{n-j} \quad(1 \leq j \leq n-1) .
$$

Based on this, Theorem 2.2 can be given the following short proof. Let $\mathbf{u} \in \mathbb{R}^{n+1} \backslash\{0\}$. By Theorem 4.2, there exists $q_{0}>0$ and an $(n+1)$-system $\mathbf{P}$ on $\left[q_{0}, \infty\right)$ such that $\mathbf{P}-\mathbf{L}_{\mathbf{u}}$ is bounded on $\left[q_{0}, \infty\right)$. For such a choice of $\mathbf{P}$, we have

$$
\underline{\psi}_{j}(\mathbf{u})=\liminf _{q \rightarrow \infty} \psi_{j}\left(q^{-1} \mathbf{P}(q)\right) \quad(1 \leq j \leq n) .
$$


Since $q^{-1} \mathbf{P}(q)$ belongs to $\bar{\Delta}^{(n+1)}$ for each $q \geq q_{0}$, the inequalities (6.2) apply to these points. They imply that

$$
0 \leq \frac{\underline{\psi}_{j}(\mathbf{u})}{j} \leq \frac{\underline{\psi}_{j+1}(\mathbf{u})}{j+1} \quad \text { and } \quad 0 \leq \frac{1-\underline{\psi}_{j}(\mathbf{u})}{n+1-j} \leq \frac{1-\underline{\psi}_{j+1}(\mathbf{u})}{n-j} \quad(1 \leq j \leq n-1),
$$

and the conclusion follows using Proposition 3.1 to translate these estimates in terms of $\omega_{0}(\mathbf{u}), \ldots, \omega_{n-1}(\mathbf{u})$.

\section{REFERENCES}

[1] Y. Bugeaud and M. Laurent, On transfer inequalities in Diophantine approximation II, Math. Z. 265 (2010), 249-262.

[2] L. G. P. Dirichlet, Verallgemeinerung eines Satzes aus der Lehre von Kettenbrüchen nebst einigen Anwendungen auf die Theorie der Zahlen, SBer. Kgl. Preuss. Akad. Wiss. Berlin, 1842, 93-95; reproduced in G. Lejeune Dirichlet's Werke, vol. 1, Cambridge U. Press (2012), 633-638.

[3] P. M. Gruber and C. G. Lekkerkerker, Geometry of numbers, North-Holland, 1987.

[4] V. Jarník, Zum Khintchineschen Übertragungssatz, Trav. Inst. Math. Tbilissi 3 (1938), 193-212.

[5] A. Y. Khintchine, Zur metrischen Theorie der diophantischen Approximationen, Math. Z. 24 (1926), $706-714$.

[6] A. Y. Khintchine, Über eine Klasse linearer diophantischer Approximationen, Rendiconti Circ. Mat. Palermo 50 (1926), 170-195.

[7] M. Laurent, Exponents of Diophantine approximation in dimension two, Can. J. Math. 61 (2009), $165-189$

[8] M. Laurent, On transfer inequalities in Diophantine approximation, in: Analytic Number Theory, Essays in honour of Klaus Roth, Cambridge U. Press (2009), 306-314.

[9] K. Mahler, Neuer Beweis eines Satz von A. Khintchine, Mat. Sbornik 43 (1936), 961-962.

[10] D. Roy, On Schmidt and Summerer parametric geometry of numbers, Ann. of Math. 182 (2015), 739786.

[11] W. M. Schmidt, On heights of algebraic subspaces and Diophantine approximations, Ann. of Math. 85 (1967), 430-472.

[12] W. M. Schmidt and L. Summerer, Parametric geometry of numbers and applications, Acta Arith. 140 (2009), 67-91.

[13] W. M. Schmidt and L. Summerer, Diophantine approximation and parametric geometry of numbers, Monatsh. Math. 169 (2013), 51-104.

Département de Mathématiques, Université d'Ottawa, 585 King Edward, Ottawa, Ontario K1N 6N5, CANADA

E-mail address: droy@uottawa.ca 